\title{
Rendimento e viabilidade da extração de hipófise de jundiá (Rhamdia quelen)
}

\author{
Silver catfish (Rhamdia quelen) pituitary yield and viability
}

\author{
Carine de Vargas Colpo ${ }^{\mathrm{I}}$ Mauro Alves da Cunha ${ }^{\mathrm{I}}$ Tanise dos Santos Medeiros ${ }^{\mathrm{I}}$ \\ Bernardo Baldisserotto ${ }^{\mathrm{I}^{*}}$
}

\section{RESUMO}

\begin{abstract}
O extrato hipofisário é provavelmente o indutor hormonal para desova e espermiação mais utilizado em piscicultura. A possibilidade de o produtor ter uma nova fonte de renda motivou a execução deste trabalho. O objetivo foi verificar o rendimento da hipófise no jundiá (Rhamdia quelen), em função do peso, e analisar a viabilidade econômica de sua extração. Exemplares $(n=116)$ com pesos entre 158-1.543g foram abatidos para extração da hipófise. $O$ índice gonadossomático (IGS) também foi calculado para verificar sua relação com o peso da hipófise. A hipófise apresentou relação significativa com o peso dos jundiás, mas não com o IGS. A extração da hipófise é mais uma opção de renda para a cadeia produtiva dessa espécie.
\end{abstract}

Palavras-chave: pituitária, extrato hipofisário, rendimento econômico em piscicultura.

\section{ABSTRACT}

The pituitary extract is probably the most used hormonal inducer to spawning and spermiation in fish culture. The possibility of a new income source for the fish farmer was the reason of this study. The aim of the present study was to verify the yield of the pituitary in silver catfish (Rhamdia quelen) as a function of weight and to analyze the economical viability of its extraction. Specimens $(n=116)$ with $158-1543 \mathrm{~g}$ were sacrificed for pituitary extraction. The gonadosomatic index (GSI) was also calculated to verify its relationship with the pituitary weight. The pituitary showed a significant relationship with silver catfish weight, but not with the GSI. The extraction of the pituitary is another profitable option to silver catfish productive market.

Key words: hypophysis, pituitary extract, fish culture income.
Muitas espécies nativas brasileiras utilizadas em piscicultura são reofílicas (GODINHO, 2007). Existe a possibilidade de estimular a reprodução desses peixes migradores através da indução ambiental, mas a complexidade dos mecanismos de controle do desenvolvimento gonadal e do comportamento reprodutivo dificulta muito a simulação das condições ideais para reprodução em cativeiro (ZANIBONI FILHO \&WEINGARTNER, 2007).

Nos peixes, a hipófise produz as gonadotrofinas I e II, as quais estimulam as gônadas para a desova e espermiação (BALDISSEROTTO, 2009). A aplicação do extrato bruto da hipófise (hipofisação) de peixes maduros continua sendo a técnica mais utilizada para a indução hormonal da maturação final dos peixes migradores brasileiros (ZANIBONI FILHO \&WEINGARTNER, 2007).

Os elevados preços das hipófises de carpa e de salmão nos mercados nacional e internacional estimularam a realização de trabalhos com hipófises de frango, rã e coelho, com bons resultados na indução à desova e espermiação nos dois primeiros e variáveis com hipófise de coelho (STREIT JR. et al., 2003, 2005; NAVARRO et al., 2007). Outra opção testada, a urófise de peixes, não rendeu bons resultados (BEHR et al., 2000). Buscando outras alternativas, verificou-se também o rendimento da hipófise de voga (Cyphocharax voga), uma espécie nativa e comercializada na região Sul do Brasil (MARTINS et al., 2008).

'Departamento de Fisiologia e Farmacologia, Universidade Federal de Santa Maria (UFSM), 97105-900, Santa Maria, RS, Brasil. E-mail: bbaldisserotto@ hotmail.com.*Autor para correspondência. 
Com o crescimento da produção de pescado e do alto valor atribuído à hipófise, cerca de $\mathrm{R} \$ 1.500,00$ grama $^{-1}, \mathrm{o}$ objetivo do presente trabalho foi verificar o rendimento da hipófise no jundiá, Rhamdia quelen, em função do peso e verificar a viabilidade econômica de sua extração para venda pelo piscicultor.

Cento e dezesseis exemplares machos e fêmeas de jundiá com peso entre 158 e $1.543 \mathrm{~g}$ foram obtidos do Laboratório de Piscicultura da Universidade Federal de Santa Maria e da piscicultura Cielo (NãoMe-Toque, RS). Os peixes foram transportados para o Laboratório de Fisiologia de Peixes e imediatamente sacrificados por secção da medula espinhal. Os jundiás foram então pesados, o crânio do peixe foi aberto com um alicate de corte e retirou-se a hipófise com uma pinça, a qual foi pesada individualmente (peso da hipófise fresca) em balança analítica com precisão de $1,0 \mathrm{mg}$. As hipófises coletadas foram armazenadas em acetona, a qual foi trocada três vezes durante uma semana antes de nova pesagem.

As gônadas foram retiradas para identificação do sexo e pesadas para posterior cálculo do índice gonadossomático (IGS): IGS = (peso gônada/ peso peixe) x 100. O cálculo das relações peso da hipófise fresca e peso total ou IGS do jundiá foi efetuado através do programa Sigma Plot versão 11.

O peso total de 56 hipófises frescas foi $328 \mathrm{mg}$ e, após armazenamento em acetona e exposição ao ar por $5 \mathrm{~min}$, foi $68 \mathrm{mg}$, ou seja, o peso da hipófise desidratada em acetona é $20,73 \%$ do peso fresco original. A retirada das hipófises de 56 exemplares levou $4,5 \mathrm{~h}$ de trabalho. $\mathrm{O}$ peso do peixe teve uma relação linear positiva com o peso da hipófise, isto é, quanto maior o peso, maior a hipófise (Figura 1). Já o IGS variou de 1,12 a 18,24 e não teve relação significativa com o peso das hipófises.

Voga machos e fêmeas com pesos médio de 122,6 e $150,7 \mathrm{~g}$, respectivamente, rendem hipófises desidratadas em acetona com 0,32 e $0,41 \mathrm{mg}$ respectivamente, o que levou os autores a considerar que a hipófise das fêmeas é significativamente mais pesada que a dos machos (MARTINS et al., 2008). No entanto, utilizando a equação que relaciona o peso total do jundiá com o peso da hipófise, verifica-se que exemplares com 122,6 e 150,7g rendem hipófises desidratadas em acetona com 0,36 e $0,47 \mathrm{mg}$, respectivamente. Pesos semelhantes aos encontrados para voga por MARTINS et al. (2008), ou seja, provavelmente a diferença encontrada por MARTINS et al. (2008) para machos e fêmeas de voga está relacionada com o peso dos exemplares e não com o sexo.

A relação do peso da hipófise seca de bacalhau (Gadus morhua) com o peso total é $0,84 \mathrm{mg}$ $\mathrm{kg}^{-1}$ (WOODHEAD, 1971). Este valor é menor que o correspondente para hipófise de jundiá com $1 \mathrm{~kg}$ seca

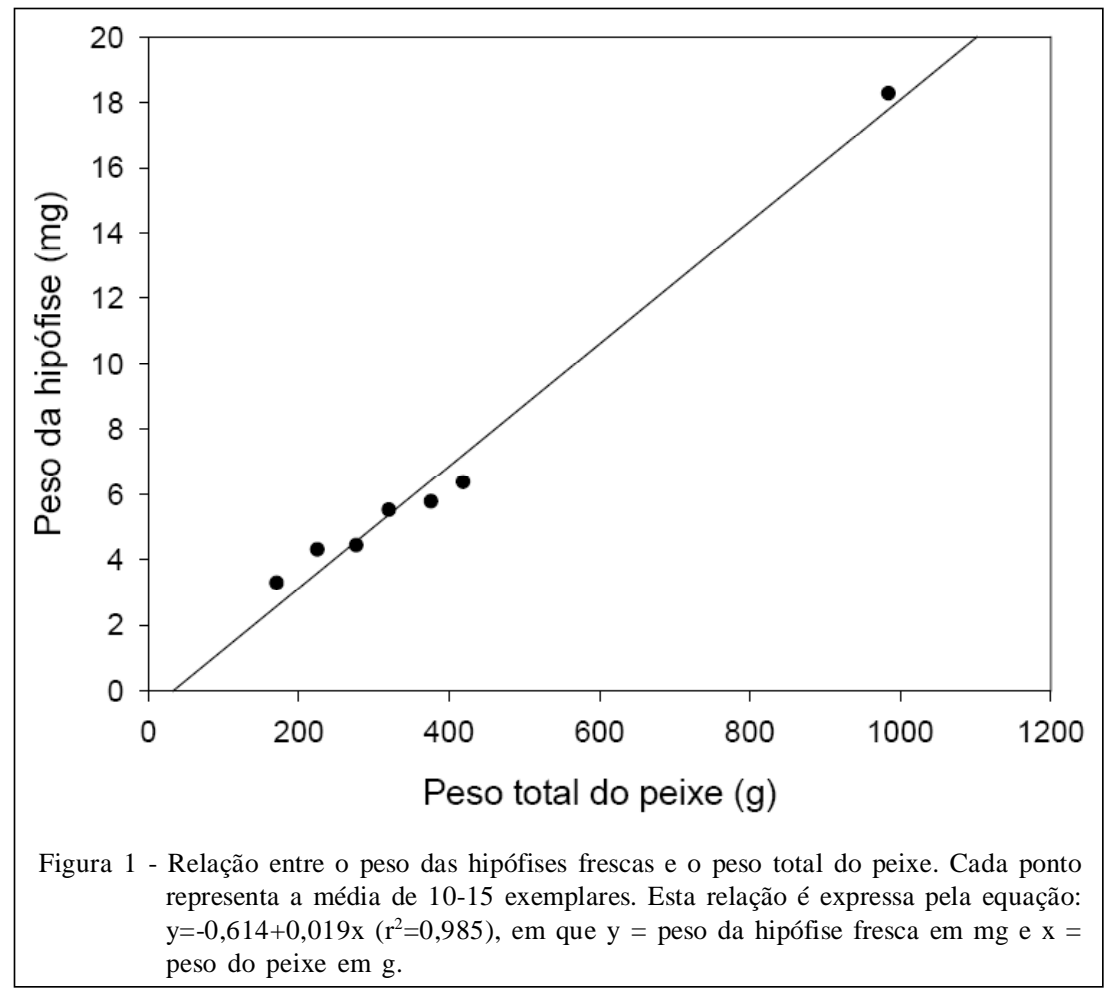

Ciência Rural, v.41, n.5, mai, 2011. 
em acetona $(3,81 \mathrm{mg})$, provavelmente porque no referido estudo a hipófise foi seca em estufa.

Quanto maior o jundiá, maior será a possibilidade de venda da carne e de agregar valor extraindo a hipófise. Em jundiás com 700g, o peso da hipófise fresca e seca em acetona fica em 12,69 e 2,63mg, respectivamente. Para exemplares com $1.000 \mathrm{~g}$, a hipófise fresca e seca pesaria 18,38 e 3,81mg, respectivamente. Portanto, para obter $1 \mathrm{~g}$ de hipófise seca em acetona para venda, seria necessário em torno de 380 jundiás de $700 \mathrm{~g}$ ou 262 exemplares de $1.000 \mathrm{~g}$.

Para obtenção de $1 \mathrm{~g}$ de hipófise seca em acetona, seriam necessários 30,54 e 21,05h de trabalho para exemplares de 700 e $1.000 \mathrm{~g}$, respectivamente. Levando em consideração o salário mínimo mensal de $\mathrm{R} \$ 511,29$ para 40 horas semanais ou 160 horas mensais no Rio Grande do Sul em julho de 2009. O valor de mãode-obra seria $\mathrm{R} \$ 3,20$ por hora.

Para obtenção de $1 \mathrm{~g}$ de hipófise seca em acetona de jundiá, o custo de mão-de-obra seria $\mathrm{R} \$$ 97,79 e R \$77,36, respectivamente, utilizando exemplares de 700 e $1.000 \mathrm{~g}$. Sendo o valor de mercado de $1 \mathrm{~g}$ de hipófise R\$ $1.500,00$, pode-se obter um lucro de R\$ $1.402,21$ e R\$1.422,64 para exemplares de 700 e $1.000 \mathrm{~g}$, respectivamente. A extração da hipófise do jundiá é uma fonte alternativa para a cadeia produtiva da espécie.

\section{AGRADECIMENTOS}

C. Colpo e B. Baldisserotto receberam bolsa do Programa Institucional de Bolsas de Iniciação Científica (PIBIC) e produtividade do Conselho Nacional de desenvolvimento Científico e Tecnológico (CNPq), M.A. Cunha e T.S. Medeiros de Doutorado e Mestrado da Coordenação de Aperfeiçoamento de Pessoal de Nível Superior (CAPES).

\section{REFERÊNCIAS}

BALDISSEROTTO, B. Fisiologia de peixes aplicada à piscicultura. 2.ed. Santa Maria: Universidade Federalde Santa Maria, 2009. 350p.

BEHR, E.R. et al. Urophysial and pituitary extracts for spawning induction in teleosts. Ciência Rural, v.30, p.897-898, 2000. Disponível em: 〈http://www.scielo.br/pdf/cr/v30n5/a27v30n5.pdf>. Acesso em: 15 mar 2011. doi: 10.1590/S010384782000000500027 .
GODINHO, H.P. Estratégias reprodutivas de peixes aplicadas à aqüicultura: bases para o desenvolvimento de tecnologias de produção. Revista Brasileira de Reprodução Animal, v.31, p.351-360, 2007. Disponível em: <http://www.cbra.org.br/ pages/publicacoes/rbra/download/351.pdf > . Acesso em: 20 ago. 2009.

MARTINS, C.R. et al. Extração e rendimento da hipófise da Cyphocharax voga em relação à maturação gonadal e ao sexo. Archivos de Zootecnia v.57, n.218, p.284-286, 2008. Disponível em: <http://redalyc.uaemex.mx/redalyc/pdf/495/ 49515018022.pdf>. Acesso em 15 mar. 2011.

NAVARRO, R.D. et al. Reprodução induzida de curimbatá (Prochilodus affinis) com uso de extrato bruto hipofisário de rã touro (Rana catesbeiana). Zootecnia Tropical, v.25, n.2, p.143-147, 2007. Disponível em: <http://sian.inia.gob.ve/ repositorio/revistas_ci/ZootecniaTropical/zt2502/arti/ navarro.htm>. Acesso em: 15 mar. 2011.

STREIT JR., D.P. et al. Estudo comparativo da indução hormonal da espermiação em piavuçu (Leporinus macrocephalus) com extrato de hipófise de frango, coelho e carpa. Acta Scientiarum, Animal Sciences, v.25, n.2, p.261266, 2003. Disponível em: <http://periodicos.uem.br/ojs/ index.php/ActaSciAnimSci/article/view/1996/1399>. Acesso em: 15 mar. 2011. doi: 10.4025/actascianimsci.v25i2.1996.

STREIT JR., D.P. et al. Effects of three different sources of pituitary extract on gonadal inducer in male and female pacu (Piaractus mesopotamicus). Acta Scientiarum, Animal Sciences, v.27, n.4, p.439-447, 2005. Disponível em: <http:/ /periodicos.uem.br/ojs/index.php/ActaSciAnimSci/article/ viewFile/1146/602>. Acesso em: 15 mar. 2011. doi: 10.4025/ actascianimsci.v27i4.1146.

WOODHEAD, P.M.J. Relationship of pituitary size to body weight in cod, Gadus morhua. General and Comparative Endocrinology, v.16, n.1, p.160-162, 1971. Disponível em: $<\mathrm{ht} \mathrm{t} \mathrm{p} \mathrm{:} \mathrm{/} \mathrm{/} \mathrm{w} \mathrm{w} \mathrm{w.} \mathrm{s} \mathrm{c} \mathrm{i} \mathrm{e} \mathrm{n} \mathrm{c} \mathrm{e} \mathrm{d} \mathrm{i} \mathrm{rect.} \mathrm{c} \mathrm{o} \mathrm{m} \mathrm{/}$ science?_ob=ArticleURL\&_udi=B 6W G0-4DXB6J7 2N\&_user=687358\&_coverDate $=02 \% 2 \mathrm{~F} 28 \% 2 \mathrm{~F} 1971 \& \_$rdoc $=1 \& \_\mathrm{fm}$ $\mathrm{t}=\mathrm{high} \&$ orig=gateway\&_origin=gateway\&_sort $=\mathrm{d} \&$ docanchor $=\&$ view=c\&_searchStrId=1696557055\&_rerunOrigin $=$ google \&_acct $=$ C000037899\&_version=1 \&_urlVersion=0\&_userid $=687358 \& \mathrm{md}$ $5=21 \mathrm{da} 6467436 \mathrm{e} 02 \mathrm{afb} 0964 \mathrm{~b} 6 \mathrm{c} 7 \mathrm{~d} 063 \mathrm{bf} 7 \&$ searchtype $=\mathrm{a}$. Acesso em: 15 mar. 2011. doi:10.1016/0016-6480(71)90219-X.

ZANIBONI FILHO, E.; WEINGARTNER, M. Técnicas de indução da reprodução de peixes migradores. Revista Brasileira de Reprodução Animal, v.31, p.367-373, 2007. Disponível em: <http://www.cbra.org.br/pages/publicacoes/rbra/download/ 367.pdf>. Acesso em: 15 mar. 2011. 